\title{
Longitudinal Relations of Parental Influences and Adolescent Career Aspirations and Actions in a Collectivist Society
}

\author{
Dian Ratna Sawitri, Peter Creed, and Melanie Zimmer-Gembeck \\ School of Applied Psychology \\ Griffith University
}

\begin{abstract}
Using social cognitive career theory as a framework, we examined the longitudinal effects of proximal parental contextual influences on career aspirations and actions in a collectivist context. We used a sample of 954 Indonesian high school students, and measured parental career expectations, adolescent-parent career congruence, self-efficacy, outcome expectations, career aspirations, planning, and exploration, twice, six months apart. The best fitting model was reciprocal, with the results showing that parental career expectations predicted subsequent career aspirations and planning, and aspirations and congruence with parents regarding career matters predicted future exploration. Self-efficacy and outcome expectations were reciprocally related over time. Self-efficacy served as an across-time predictor of both parental contextual variables. Career exploration predicted future self-efficacy and planning predicted later outcome expectations.
\end{abstract}

Keywords: social-cognitive career theory; parental career expectations; adolescent-parent career congruence; career aspirations; career actions of planning and exploration; collectivist society 
The social cognitive career theory choice model (Lent, Brown, \& Hackett, 1994) has been tested largely in Western societies, where it has been used to explain the associations between proximal contextual variables and career goals and actions (e.g., Lent et al., 2003; Rogers, Creed, \& Glendon, 2008). In these studies, supports and barriers have been the most widely tested variables during the active stages of career decision-making (i.e., when individuals are considering careerrelated options and are making career decisions). These proximal contextual variables have been linked cross-sectionally to career goals and actions in specific subject matter areas (i.e., science, computing, engineering, and mathematics; Lent et al., 2001; Lent et al., 2003), more generally (e.g., career planning and exploration; Betz \& Voyten, 1997; Rogers et al., 2008), as well as with specific populations, such as girls and women (e.g., Flores \& O’Brien, 2002; Schaefers, Epperson, \& Nauta, 1997) and ethnic minority groups (e.g., Ojeda \& Flores, 2008; Tang, Fouad, \& Smith, 1999).

More recently, proximal contextual variables were investigated longitudinally in relation to college students' career actions (Lent et al., 2008; Lent, Sheu, Gloster, \& Wilkins, 2010). However, with the exception of a few studies (e.g., Rogers \& Creed, 2011), there has been little research examining the longitudinal relationships among social cognitive career variables during active stages of career decision-making, such as during the high school years (Lent et al., 2008). Moreover, little is known about how proximal contextual variables might operate in non-Western cultural contexts, where manifestations of supports and barriers salient for these contexts (e.g., congruence with parents regarding career matters) have not been tested (Lent et al., 2003). Finally, although both Bandura’s (1999, 2000) and Lent et al.’s (1994) models have been used to explain how proximal contextual variables operate in relation to goals and actions (e.g., Lent et al., 2001; Lent et al., 2003), they have not been tested longitudinally using participants from a collectivist society. The current study was designed to test the longitudinal influences of proximal parental contextual variables (operationalized as perceived parental career expectations and perceived congruence with 
parents regarding career matters) on high school students' career goals (i.e., aspirations) and actions (i.e., planning and exploration) in a collectivist culture. We used the social cognitive career theory as the general framework, which allowed us to test an integrated model of Bandura's $(1999,2000)$ and Lent et al.’s (1994) proposals.

\section{Proximal Contextual Influences from a Social Cognitive Perspective}

Originally derived from the general social cognitive theory (Bandura, 1986), social cognitive career theory (Lent et al., 1994) proposed that self-efficacy, outcome expectations, and goals are the essential agency variables that enable individuals to develop personal control over their career development process by affecting the development of career interest and choice behaviours. Additionally, contextual variables, physical attributes, and learning experiences also contribute to this process. Since successful career choice behaviours are not entirely determined by competencies in formulating and actualising goals, but are influenced also by environmental factors perceived to be significant in the pursuit of goals (Lent et al., 2000), a variety of contextual variables have been assessed by career researchers. These environmental influences have been tested as distal contextual variables (i.e., variables which shape the learning experiences that stimulate individuals to develop self-efficacy and positive outcome expectations, such as parents' education level and role modeling; Flores \& O’Brien, 2002; Ojeda \& Flores, 2008), and tested as proximal contextual influences (i.e., that affect individuals during active phases of career decision making, such as supports and barriers; Lent et al., 2001; Lent et al., 2003).

While many proximal contextual influences have been assessed, a number of researchers have highlighted two views regarding the way these variables might relate to goals and actions, especially within the social cognitive career choice model. The social cognitive career theory proposes direct paths to goals and actions (Lent et al., 1994), while the general social cognitive theory suggests indirect paths to actions via self-efficacy and goals (Bandura, 1999, 2000). Prior cross-sectional studies have compared and incorporated both views and investigated the role of 
proximal contextual variables with several kinds of goals, such as choice goals, educational goals, occupational considerations, and many types of actions, such as choice stability and persistence (Lent et al., 2001; Lent et al., 2003; Lent et al., 2005; Lent et al., 2011). Most studies have demonstrated support for indirect paths to goals and actions, primarily via self-efficacy (Lent et al., 2001; Lent et al., 2003), although several studies have found evidence for direct and indirect paths to goals (Lent et al., 2005; Lent et al., 2011).

Proximal contextual variables also have been integrated into longitudinal studies to explain career actions such as career planning, career exploration, and goal persistence (e.g., Lent et al., 2010; Rogers \& Creed, 2011). Rogers and Creed (2011) identified a significant across-time relationship between support and career exploration in high school students. While the application of the social cognitive career choice model outside the subject matter field has been tested (e.g., to predict career actions of planning and exploration), the bi-directional relationships among the variables in the longitudinal model have been unexplored. The examination of the reciprocal relationship is important because, from the agentic socio-cognitive perspective, individuals are not only reactive to external influences, but are also proactive and able to self-regulate (Bandura, 1999). In other words, individuals are "products" as well as "producers" of their environments (Bandura, 2000). A previous longitudinal study by Lent et al. (2010) favoured a bi-directional model over a unidirectional model to explain the longitudinal relationships among the variables in the social cognitive career choice model. However, the theoretically plausible reverse paths from goal persistence to future outcome expectations were untested, and similar to most earlier studies, this study tested the social cognitive choice model on college students within a specific subject matter domain, rather than more generally.

\section{Career-Related Contextual Influences in a Collectivist Society}


The unique features of collectivist cultures have attracted researchers to investigate how contextual variables influence adolescents’ career behaviours in those settings. In collectivist cultures, personal agency is located not only within the individual, but also in affirmative relationships with significant others, mainly parents (Cross \& Markus, 1999; Oettingen \& Zosuls, 2006). For example, in cultures with a large power distance index, the less powerful members of an institution, in this case, children in the family, expect and accept an unequal distribution of power from the more powerful members (i.e., parents; Hofstede \& Hofstede, 2005). Therefore, obedience to parents is usually highlighted. As parents serve as potential sources of their children's efficacy beliefs and have a considerable influence on their children's choices (Oettingen \& Zosuls, 2006), it is unusual for children to ignore parental expectations and input when formulating their career aspirations and engaging in career-related actions (Hardin, Leong, \& Osipow, 2001). While person variables (e.g., personality), self-efficacy, and outcome expectations serve as strong predictors of career goals and actions in Western contexts (e.g., Rogers et al., 2008), studies with individuals from collectivist cultures have shown that parental involvement and self-efficacy are associated directly with career choice, but personal interests are not (e.g., Tang et al., 1999).

We examined two important contextual variables that have been found to affect individual career progress. The first is parental career expectations, which can impinge upon decisions related to educational and occupational aspirations, occupational choice, and level of commitment and effort (Fouad et al., 2008; Shea, Ma, \& Yeh, 2007). Parental expectations are perceived as most facilitative by adolescents when they are congruent with the child's own expectations. For example, adolescents who believed that they met their parents' expectations were less vulnerable to stress (Wang \& Heppner, 2002) and experienced fewer career difficulties (Leung et al., 2011).

The second parental contextual variable is parental support, which includes encouragement, instrumental assistance, modelling desired behaviours, and emotional backing. Both cross-sectional and longitudinal studies with participants from collectivist cultures have demonstrated that parental 
support affects children’s career behaviours (e.g., Cheung \& Arnold, 2010; Garcia, Restubog, Toledano, Tolentino, \& Rafferty, 2012). For example, Restubog, Florentino, and Garcia (2010) found that parental support and the number of career counselling sessions received were related indirectly to career decidedness via self-efficacy in the six months following counselling, and, in turn, self-efficacy was found to indirectly predict subsequent persistence via career decidedness. Similar to parental expectations, support from parents has the desired effect mainly when the action is perceived by their children to be supportive (Garcia et al., 2012). This means that the anticipated consequences of parental career-related actions will be achieved when these actions are subjectively interpreted in the same way by both parties (Lent et al., 2000). This congruence between children and their parents has been operationalized in different studies as adjustment with parental approval, living-up to parental expectations, and adolescent-parent career congruence (e.g., Leong \& Serafica, 1995; Leung et al., 2011).

\section{The Present Study}

The present study examined whether a standard causal model or a reciprocal model showed the best fit to explain the longitudinal associations between selected proximal, parental contextual variables (perceived parental career expectations and perceived adolescent-parent career congruence) and career aspirations and the career actions of planning and exploration. We utilized a large sample of adolescents from a collectivist society and used the social cognitive career theory as a framework. The study built on previous research in several notable ways: (a) we used the social cognitive career theory perspective to explain both career goals and actions, (b) we involved a large sample of Indonesian high school students, as this country has been categorised as a collectivist country (Hofstede \& Hofstede, 2005), (c) we incorporated Lent’s (1994) and Bandura’s (1999, 2000) views on how proximal contextual variables should operate, (d) we tested these relationships longitudinally, at two time points during an active period of decision making, at Time 1 (T1), when students were considering their education or career-related options, and at Time 2 (T2), after they 
had made decisions about these options, and (e) we tested all theoretically plausible paths based on the cyclical nature of the social cognitive career variables.

In the across-time, standard-causal model, we expected that (a) T1 self-efficacy would predict T2 outcome expectations, (b) T1 self-efficacy and outcome expectations would predict T2 aspirations, planning, and exploration, and (c) T1 aspirations would predict T2 planning and exploration (Lent et al., 1994). We also expected that both T1 parental variables would predict (d) T2 aspirations (Bandura, 1999, 2000; Lent et al., 1994), (e) T2 planning and exploration (Lent et al., 1994), and (f) T2 self-efficacy (Bandura, 1999, 2000). The hypothesised standard-causal model is illustrated in Figure 1.

Further, we tested a reciprocal model that contained all paths in the standard causal model, as well as several reverse-direction paths, where (g) T1 outcome expectations predicted T2 selfefficacy, as hypothesised by Lent et al. (2008), (h) T1 self-efficacy predicted both T2 parental variables, as proposed by Lent et al. (2000), as individuals who are more efficacious in a particular domain tend to view contextual variables as having more positive influences on their domain-related progress, and (i) T1 planning and exploration predicted T2 self-efficacy and outcome expectations, as suggested by Lent (2005), as the effect of goal progress on self-efficacy and outcome expectations is reciprocal.

\section{Method}

\section{Participants}

Participants at T1 were 1056 Grade 10 students from several schools situated in urban areas in a medium sized city in Central Java, Indonesia. Students largely had low to middle socioeconomic backgrounds. The sample was $54.4 \%$ female, and the age range was 14.4 to 18.8 years ( $M$ $=15.93, S D=.51$ ), although the vast majority of students (approximately $90 \%$ ) were in the age range of 15.3 to 16.6 years. On a self-reported measure of school achievement, seven students (.7\%) rated their level to be “below average”, 527 (49.6\%) rated themselves to be “average”, 522 (49.2\%) to be 
“above average”, and six (.6\%) to be “well above average”. Nine students (.9\%) reported that their socio-economic status was “much worse” than their peers, 59 (6.2\%) reported “a little worse”, 590 (61.8\%) reported “about the same”, 257 (26.9\%) reported “a little better”, and 39 (4.1\%) indicated “much better” condition. Of the students, 120 (11.4\%) reported that they were working while studying. At T2, six months later, 954 Grade 11 students (55.8\% female; age range 14.9 to 18.7 years, $M=16.43, S D=.49$ ) responded to a second survey (response rate $=90 \%$ ).

\section{Materials}

At both data collection points, the survey packets contained measures of parental career expectations, adolescent-parent career congruence, self-efficacy, outcome expectations, career aspirations, and the career actions of planning and exploration. For consistency for the students across scales, we used 6-point Likert-like response formats at both times. This number of options was recommended by Weng (2004) when an even number of response options are used. Thus, the 5point response-formats for the self-efficacy, career aspirations, career planning, and career exploration scales, and the 4-point response format for the outcome expectations scale, were elevated to 6-point response formats.

Parental career expectations. We assessed students’ perceptions of parental expectations regarding their career options and academic attainment using the 9-item Perceived Parental Expectation Subscale (Academic Achievement Domain) from the Living up to Parental Expectations Scale (Wang \& Heppner, 2002). On a 6-point Likert-type scale $(1=$ not at all expected and $6=$ very strongly expected), students responded to items such as "Your parents expect you to pursue their ideal careers (e.g., doctor, teacher...), how strong do you perceive this from your parents?” Higher scores reflect stronger perceived parental expectations for achieving academic and career success. The test-retest coefficient over a 4-week interval was reported to be moderate and alphas of the Perceived Parental Expectation Subscale-Academic Domain were reported as greater than .80 
(Leung, Hou, Gati, \& Li, 2011; Wang \& Heppner, 2002). Validity evidence was demonstrated through a factor analysis and correlations with related variables (Wang \& Heppner, 2002).

Adolescent-parent career congruence. We used the 12-item Adolescent-Parent Career Congruence Scale (XXX, 2013) to measure complementary congruence (i.e., adolescent perceptions that parents are facilitative in their career progress and are satisfied with their career-related actions and progress) and supplementary congruence (i.e., adolescent perceptions that parents have parallel career values, interests, aspirations, and plans). On a 6-point Likert-type scale ( 1 = strongly disagree and 6 = strongly agree), students responded to items such as "The progress I have made towards my career goals makes my parents happy.” Higher scores reflect more perceived career congruence with parents. Alpha was reported as .87 (XXX, 2013). Evidence of validity is supported by positive correlations with measures of well-being, living-up-to parental expectations, and parental support (XXX, 2013).

Self-efficacy. We assessed students’ level of efficacy regarding their ability to carry out career tasks using the 12-item Career Decision-Making Self-Efficacy Subscale of The Middle School Self-Efficacy Scale (Fouad, Smith, \& Enochs, 1997). Students indicated their responses to items such as "How confident are you that you could select one occupation from a list of possible occupations you are considering?” using a 6-point Likert-type scale $(1=$ not confident at all and $6=$ highly confident). Higher scores indicate stronger career decision-making self-efficacy. Previous studies have reported alpha estimates of .89 and .79, and have supported for validity by finding expected correlations with other career-related variables (Creed, Tilbury, Buys, \& Crawford, 2011; Fouad et al., 1997).

Outcome expectations. We used the 6-item Vocational Outcome Expectations Scale (McWhirter, Rasheed, \& Crothers, 2000) to assess students’ optimism regarding expected career outcomes. Students responded along a 6-point Likert-type scale $(1=$ strongly disagree and $6=$ strongly agree) to items such as “My career planning will lead to a satisfying career for me.” Higher 
scores reflect stronger career outcome expectations. Reliability coefficients of greater than .80 and a 9-week test-retest coefficient of .59 have been reported (Ali, McWhirter, \& Chronister, 2005;

McWhirter et al., 2000). The scale has shown positive correlations with the Vocational/Educational Self-Efficacy Belief Scale and Fouad and Smith’s (1996) outcome expectation scale (Ali et al., 2005; McWhirter et al., 2000).

Career aspirations. We used the 10-item Career Aspirations Scale (O'Brien, 1996) to measure students' aspirations to seek leadership positions and promotions, to manage others, and to gain further education to advantage their future careers. Along a 6-point Likert-type scale $(1=$ strongly disagree and 6 = strongly agree), students indicated their agreement to items such as "I plan on developing as an expert in my career field.” Two items, which were negatively worded, were reversed prior to calculating the total score. Higher scores reflect more ambitious career aspirations. Alphas of .74 and .84 have been reported (O’Brien, 1996; Reynolds \& Constantine, 2007). O’Brien (1996) provided support for validity by identifying expected relationships with career salience, academic achievement, negative affectivity, and occupational traditionality.

Career planning. We assessed students’ involvement in career planning using the 8-item Career Thinking and Planning Subscale of the Career Salience Scale (Greenhaus, 1971). Students indicated their level of agreement along a 6-point Likert-type scale $(1=$ strongly disagree and $6=$ strongly agree) to items such as "Planning for and succeeding in a career is my primary concern.” Higher scores indicate more involvement in career planning. This scale demonstrated reliability coefficients of .70 and .74 (Creed, Fallon, \& Hood, 2009), and showed expected correlations with career exploration, self-regulation, and job-seeking behaviour (Creed et al., 2009).

Career exploration. We measured students’ engagement in self- and environmental exploration activities during the previous three months using the Career Exploration Survey (Stumpf, Colarelli, \& Hartman, 1983). Students responded using a 6-point Likert-type scale (1 = almost never and 6 = very often) to five self-exploration questions, such as "I have been contemplating my past 
life and experiences in relation to my career.” They also responded to six specific environmental exploration questions, such as "I have sought information on specific areas of my career interest.” Higher scores on the 11 items reflect more engagement in career exploration activities. Reliability coefficient for the full scale has been reported as .90 (Hirschi, 2011), and in support of validity expected relationships with relational support, career planning, and career concerns have been found (Cheung \& Arnold, 2010; Creed et al., 2009).

\section{Translation Procedure}

We used Brislin’s (1986) forward and backward translation technique to convert the original English items into Bahasa (the Indonesian language): (a) the original items were translated into the target language by two bilingual native Indonesian speakers, and the readability of the translation was examined by two monolingual Indonesian speakers; (b) two bilingual native Indonesian speakers blindly back-translated the items into English; (c) to check the precision of meaning, the backtranslated version and the original items were then compared, and any inaccuracies were adjusted; and (d) three Indonesian high school students checked whether the final Bahasa version was easy to understand.

\section{Data Collection Procedure}

The study used a two-wave, longitudinal panel design that involved the same measures at each wave of data collection. Consistent with the previous longitudinal study by Rogers and Creed (2011), which also involved high school students, the six months interval between the two waves was considered sufficient for developmental changes to occur in both predictor and outcome variables. In the last quarter of Grade 10, students are encouraged to think about and choose a school major for the following two years. This choice has an effect on the university courses they will be able to select, and will affect other career-related trajectories. In this process, the students will also discuss their educational paths with significant others, especially parents. At the end of Grade 10, they have to select their major, although there is some opportunity to reconsider their choice before 
they actually commence Grade 11 . Therefore, the six month period between the two data collection points covered the transition period from Grade 10 to Grade 11, during which active consideration and career decision-making are undertaken.

The study was conducted with approval from the authors' university ethics committee. Prior to the T1 survey, we collected consent forms from both parents and students. Both surveys were administered in class time during school hours by the class teachers and supervised by the first author. Due to some students being away from school or participating in other school activities on the T2 data collection days, 102 students did not take part in the T2 survey. The data reported in this study constitute one part of a larger study (e.g., XXX, 2013).

\section{Results}

\section{Attrition Analysis}

We compared the students who did not complete the second wave with those who took part in both waves on the variables assessed at T1. We found no differences in terms of age $(p=.51)$, work experience $(p=.43)$, and socio-economic status $(p=.46)$. The two groups of students also did not differ on parental career expectations $(p=.16)$, congruence $(p=.52)$, self-efficacy $(p=.11)$, outcome expectations $(p=.13)$, aspirations $(p=.11)$, planning $(p=.24)$, and exploration $(p=.16)$. However, there were more boys (58.8\% vs. $44.2 \%$ ) in the drop-out group, and this group reported lower school achievement $(M=7.34$ vs. $7.47, p<.05)$, indicating some bias in the final sample that should be considered when interpreting the results.

\section{Data Management for Model Testing}

To achieve an ideal ratio of 20:1 for participants to observed variables (Kline, 1998), we used three and four-item parcels to represent the latent variables (Hau \& Marsh, 2004). As suggested by Little, Cunningham, Shahar, and Widaman (2002), we used the item-to-construct balance procedure to compose four-item parcels for adolescent-parent career congruence and self-efficacy, and to create three-item parcels for the rest of the scales. We conducted exploratory factor analyses specifying a 
single-factor solution for each of the scales and used the three or four items with the highest loadings to anchor each parcel. We then allocated items from the next group of three or four items to the individual parcels in the reverse order, and so on, until all items were distributed.

\section{Model Testing}

Prior to assessing the main models, we examined the measurement model. We did not control for any demographic variables in the analyses as there were insignificant or negligible relationships with age, gender, level of achievement, socioeconomic status, and work experience $(\mathrm{T} 1$ range $=-.08$ to .15 ; $\mathrm{T} 2$ range $=.09$ to .16 ). We then tested (a) a baseline model consisting of stability and synchronous effects, (b) a standard-causal model, incorporating all paths in the baseline model and the hypothesised lagged paths from the T1 to T2 variables, and (c) a reciprocal model, involving all paths in the standard-causal model and the hypothesized reverse-direction paths. We tested all models using maximum likelihood estimation in the AMOS statistical package and examined model fit using chi-square $\left(\chi^{2}\right)$, Comparative Fit Index (CFI), Tucker-Lewis Index (TLI), and the root mean square error of approximation (RMSEA). As suggested by Hair, Black, Babin, and Anderson (2010), with 954 participants and 46 observed variables, a significant $\chi^{2}$, CFI and TLI values more than .90 and an RMSEA more than .07 indicate a good fit. Chi square values are sensitive to sample size; thus, we also used the $\chi^{2} / d f$ statistic, with values less than 3 indicating a satisfactory fit. We then used the $\chi^{2}$ difference statistic $\left(\Delta \chi^{2}\right)$ to assess whether the standard causal model and the reciprocal model were significantly different from the baseline model (the $\Delta \chi^{2}$ statistic can be used to assess which of two competing nested models is a better fit to the data; if the two models do not differ, the more parsimonious model would be selected; Schumacker \& Lomax, 2010).

The measurement model yielded satisfactory fit statistics, $\chi^{2}(885)=2239.44, p<.001, \chi^{2} / d f=$ 2.53, CFI $=.94, \mathrm{TLI}=.93$, RMSEA $=.04$, with factor loadings ranging from .55 to .87 . Table 1 presents the correlations among variables, means, standard deviations, and alphas. 
The baseline model produced satisfactory fit statistics, $\chi^{2}(905)=2030.58(p<.001), \chi^{2} / d f=$ $2.24, \mathrm{CFI}=.95, \mathrm{TLI}=.94, \mathrm{RMSEA}=.04$. The stability of the study variables over the six month period was indicated by the autoregressive path values, which ranged from .50 to .63 (all $p<.0012$ ).

The standard-causal model also demonstrated a satisfactory fit, with $\chi^{2}(888)=1975.18(p<$ $.001), \chi^{2} / d f=2.22, \mathrm{CFI}=.95, \mathrm{TLI}=.94, \mathrm{RMSEA}=.03$. Based on the $\Delta \chi^{2}$ statistic, the standard causal model was a significantly better fit than the baseline model, $\Delta \chi^{2}(17)=55.40, p<.001$. In the standard causal model, T1 parental expectations predicted T2 aspirations $(\beta=.10, p<.05)$ and T2 planning $(\beta=.08, p<.05)$, T1 congruence predicted T2 exploration $(\beta=.10, p<.05)$, T1 selfefficacy predicted T2 outcome expectations $(\beta=.26, p<.001)$, and T1 outcome expectations ( $\beta=$ $.13, p<.05)$ and T1 aspirations $(\beta=.12, p<.01)$ predicted T2 exploration. Path values in the standard-causal model are depicted in Table 2.

The reciprocal model, which contained all paths in the standard causal model (see Figure 1), as well as seven reverse-direction paths (i.e., T1 outcome expectations $\rightarrow$ T2 self-efficacy, T1 selfefficacy $\rightarrow$ T2 congruence and T2 parental expectations, T1 planning $\rightarrow$ T2 self-efficacy and T2 outcome expectations, and T1 exploration $\rightarrow \mathrm{T} 2$ self-efficacy and T2 outcome expectations), yielded the best fit indices, $\chi^{2}(881)=1942.54(p<.001), \chi^{2} / d f=2.21$, CFI $=.95$, TLI $=.94$, RMSEA $=.04$. The reciprocal model was a significantly better fit than the baseline model, $\Delta \chi^{2}(14)=88.04, p<.001$, and additionally, was a significantly better fit than the standard-causal model, $\Delta \chi^{2}(7)=32.64, p<$ .001. Thus, the reciprocal model was accepted as the best fitting model. In the reciprocal model, T1 parental expectations predicted T2 aspirations $(\beta=.09, p<.05)$ and T2 planning $(\beta=.08, p<.05)$, T1 congruence $(\beta=.08, p<.05)$ and T1 aspirations $(\beta=.12, p<.01)$ predicted T2 exploration, and T1 self-efficacy predicted T2 outcome expectations $(\beta=.22, p<.05)$. We also found that T1 planning predicted T2 outcome expectations $(\beta=.12, p<.05)$, T1 exploration $(\beta=.12, p<01)$ and T1 outcome expectations $(\beta=.12, p<.05)$ predicted T2 self-efficacy, and T1 self-efficacy predicted T2 parental expectations $(\beta=.08, p<.05)$ and T2 congruence $(\beta=.12, p<.01)$. This final reciprocal 
model (with 11 standard causal paths and two reverse-direction paths deleted to reduce clutter) is presented in Figure 2. Path values for the reciprocal model are also illustrated in Table 2.

\section{Discussion}

This study examined the longitudinal relationships between two parental variables (adolescent perceptions of parental career expectations and adolescent-parent career congruence) and career aspirations and the career actions of planning and exploration in a sample of high school students from a collectivist society, using the social cognitive career choice model. As model fit did not vary as a function of gender or age, we reported results for boys and girls combined and for ages 13.9 to 17.7 years (T1) collapsed. We have extended previous cross-sectional (e.g., Lent et al., 2003; Rogers et al., 2008; Tang et al., 1999) and longitudinal (e.g., Lent et al., 2010; Rogers \& Creed, 2011) social cognitive career theory-based studies, by involving participants from a collectivist setting and testing both standard-causal and reciprocal models. We also expanded on earlier studies, in which parental career expectations, adolescent-parent career-related correspondences, and their effects on career-related outcomes have been identified (e.g., Garcia et al., 2012; Leung et al., 2011; Shea et al., 2007; Wang \& Heppner, 2002), by involving both parental career expectations and adolescent-parent career congruence in the model.

In line with Lent et al.’s (2010) study, the reciprocal model was found to be a better fit than the baseline and standard causal models to explain the longitudinal relationships among the constructs of interest. Our study contributes to the literature in several important ways. First, T1 parental career expectations were found to predict T2 aspirations and T2 planning, suggesting that higher perceived parental career expectations are likely to motivate students to have higher career aspirations and to engage more in career planning over time. This finding is in line with previous cross-sectional findings (e.g., Tang et al., 1999), in which parental variables were found to relate to career outcomes. These results also are in line with Lent et al.’s (1994) suggestion that cultural context is an important influence on academic and career choices. Further, the non-significant 
relationship between T1 aspirations and T2 planning suggests that perceived parental career expectations are more relevant in predicting students' future career planning rather than their own aspirations.

Second, T1 congruence and T1 aspirations were found to predict T2 exploration, suggesting that both setting high aspirations and being congruent with parents regarding career issues are important for promoting adolescents' subsequent career exploration. This finding expands the range of cross-sectional and longitudinal predictors of adolescent career exploration behaviour in collectivist cultures (e.g., Cheung \& Arnold, 2010), and extends the findings from previous studies regarding the ability of career goals to predict future actions (e.g., Lapan, Shaughnessy, \& Boggs, 1996). However, T1 parental career expectations were not related to T2 exploration. Parental expectations might affect adolescents' career goals, but the extent to which the adolescents have similar career ideas and feel that they have been supported by their parents and are meeting their parents' wishes are more facilitative of transforming their aspirations into actions in the real world. During the active period of career decision-making, these students might recognise that some aspects of parents' career expectations are not relevant to their subsequent career exploration. Additionally, some time might need to pass for these students to convert their career goals (i.e., aspirations) into goal-directed behaviours (i.e., exploration). Together with congruence, career aspirations might guide these students when engaging in later career exploration activities.

Contrary to expectations, neither T1 parental variables predicted T2 self-efficacy. This finding is inconsistent with Oettingen and Zosul's (2006) view that in collectivist cultures, especially those with a high power distance index, parents usually are an important source of efficacy information. In our study, self-efficacy at T2 was predicted by T1 exploration, showing that involvement in career exploration activities is likely to promote confidence prospectively in dealing with career-related tasks. Our finding endorses that career exploration functions as goal-directed behaviour, is indicative of performance accomplishment, and is a vital source of efficacy beliefs 
(Lent et al., 1994; Rogers et al., 2008). This suggests that the six month period of time was sufficient for these students to explore relevant career-related information. Compared with perceiving parental expectations from and congruence with their parents, what these students obtained from career exploration activities appeared to be more relevant to the development of their later efficacy beliefs. Additionally, T1 planning was found to predict T2 outcome expectations, suggesting that students with higher involvement in career planning were more likely to have stronger subsequent beliefs about expected outcomes. This finding supports the notion that goal progress (i.e., planning) has a reciprocal effect on outcome expectations (Lent, 2005), and extends previous longitudinal studies that did not test the theoretically plausible path from goal-directed behavior to later outcome expectations (Lent et al., 2008).

We found significant paths from T1 self-efficacy to T2 parental career expectations and T2 congruence. This finding suggests that, over time, students with stronger efficacy beliefs tended to perceive stronger parental career expectations and a higher degree of congruence with parents regarding career matters. These findings are consistent with the Lent et al. (2010) study, which showed that self-efficacy predicted later support. The findings also suggest that individuals with a higher level of confidence in conducting career tasks might tend to see contextual resources in a more positive way (Lent et al., 2000; Lent et al., 2010).

Further, T1 self-efficacy was found to predict T2 outcome expectations, and T1 outcome expectations also predicted T2 self-efficacy, suggesting that having stronger career confidence is likely to enhance future beliefs about expected career outcomes, and vice versa. By demonstrating the reciprocal relationship between self-efficacy and outcome expectations, these findings extend Lent et al.’s (2008) study, which had demonstrated the role of self-efficacy as an across-time predictor of outcome expectations, interests, and goals, rather than the reverse. The results also corroborate Lent et al.’s (2010) finding of a bi-directional relationship between self-efficacy and outcome expectations. 
Contrary to general social cognitive career theory hypotheses, T1 self-efficacy did not predict T2 aspirations, T2 planning, and T2 exploration. Self-efficacy has been found to be a strong predictor of career goals and actions for participants in an individualist context, both crosssectionally (e.g., Lent et al., 2005; Lent, Lent et al., 2011) and longitudinally (e.g., Lent et al., 2008; Lent et al., 2010; Rogers \& Creed, 2011). One longitudinal study with participants from a collectivist country showed that self-efficacy predicted career actions over time (Restubog et al., 2010); whereas in other cross-sectional studies the relationship between self-efficacy and career goals and actions was not significant (e.g., Kelly, Gunsalus, \& Gunsalus, 2009). Compared with their individualist counterparts, adolescents in collectivist cultures are encouraged to conform to familial norms rather than to develop their own competence in establishing career paths (Oettingen \& Zosuls, 2006). Thus, it is not surprising that parental variables at T1 are more relevant in predicting T2 career aspirations and actions than self-efficacy at T1.

Further, T1 outcome expectations did not predict T2 aspirations, T2 planning, and T2 exploration. In the Western context, the predictive ability of outcome expectations has been found to be inconsistent in relation to goals and goal-related progress (e.g., Lent et al., 2003; Lent et al., 2005); however, robust associations were evident in Lent et al.’s (2001) and Byars-Winston et al.’s (2010) cross-sectional studies and Lent et al.’s (2010) longitudinal study. In the non-Western context, the positive cross-sectional relationships between expectancy beliefs and several career outcomes were more stable (e.g., Kelly et al., 2009), while the relationships in longitudinal studies have not been examined.

Taken together, these findings suggest that in cultures that emphasise obedience to and interdependence with authority figures (i.e., parents), children's behaviours might be less informed by their efficacy beliefs and expectancy judgments (also see Oettingen \& Zosuls, 2006). Selfefficacy and expectancy beliefs can still be considered important for adolescents in collectivist cultures; however, their function may differ from that in Western countries by being influenced by 
the features of the cultural context. According to Bandura (2002), individuals create their own ways of adapting to their environment that enables them to achieve expected outcomes through agentic action. In this study, self-efficacy is more relevant to later perceived parental career expectations and congruence with parents regarding career matters, rather than to later career aspirations, planning, and exploration; whereas parental influences were more relevant in explaining these subsequent career outcomes. Finally, this was one of the few studies to date to investigate career development longitudinally in a collectivist cultural context. Our results suggest that, in this cultural context, an individual's agency (i.e., the capability to choose and manage one’s own activities to attain anticipated outcomes; Bandura, 1999) operates with reference to relationships with important others (i.e., with reference to parental expectations and perceived congruence with parents regarding career matters).

As parental contextual variables and adolescents’ social cognitive capacities and actions affect one another, it is essential to propose recommendations that will be useful for parents and children. Our results suggest that those working with adolescents should (a) help them to understand how perceived parental career expectations influence their future career aspirations and planning and how perceived career congruence with parents affects their subsequent involvement in exploring the world of work, (b) help them to recognise the career expectations that their parents have for them and the degree of career congruence they have with their parents, (c) assist them to be skillful in reconciling any career-related differences they might have with their parents, (d) encourage them to be skilled in communicating their confidence in doing various career tasks, in order to obtain more realistic expectations from parents and to fine tune the degree of congruence with them, (e) encourage them to become involved in career planning, as it raises their future perceptions of desirable outcomes, and (f) help them to actively explore the world of work, as it increases their future level of efficacy. 
It is also recommended that parents should (a) understand that their career expectations influence their children's subsequent aspirations and planning, and that more career congruence with their children influences their children's later exploratory behaviours, (b) actively facilitate their children to explore career-related information and their children’s own competencies, as this will promote their children's future career confidence, and (c) realise that their career expectations and congruence with their children are subject to change after considering their children's level of selfefficacy. In collectivist settings, it is important to note that expressions of efficacy may be somewhat muffled due to cultural beliefs than lack of self-confidence (Klassen, 2004); thus, parents' awareness of their children's confidence in conducting career-related tasks should be highlighted and their readiness to flexibly adjust their career expectations and congruence with their children needs to be encouraged and emphasised.

We tested the temporal relationships of the social cognitive choice model variables using a sample of high school students in a collectivist society. However, as the study involved students from only one country, future studies need to consider replicating the research with samples from other collectivist countries. Such replications also would allow for validity testing of the measures we used. We translated the original scales into Bahasa using standard, recommended procedures (Brislin, 1986), but the applicability of these scales in collectivist cultures needs to be assessed more widely. We tested the capacity of the social cognitive choice model to explain generic career development behaviours (i.e., career exploration and planning). Future studies should assess how well the model explains career behaviours in specific areas, such as career choices related to science, technology, engineering, and mathematics (i.e., STEM) careers. Further, since we used an interval of six months between the first and second data collections, future studies should test the model in different intervals. Finally, as we collected data on two occasions only, we have not tested the ability of one or more of the agency variables (i.e., self-efficacy, outcome expectations, aspirations) to mediate the longitudinal effects of the contextual variables on goals and actions. Future studies need 
to include more than two data collection waves to obtain a fuller picture of the dynamics of adolescent career behaviours in a collectivist cultural context.

\section{References}

Ali, S. R., McWhirter, E. H., \& Chronister, K. M. (2005). Self-efficacy and vocational outcome expectations for lower SES adolescents: A pilot study. Journal of Career Assessment, 13, 4058. doi:10.1177/1069072704270273

Bandura, A. (1986). Social foundations of thought and action: A social cognitive theory. Englewood Cliffs, NJ: Prentice Hall.

Bandura, A. (1999). Social cognitive theory of personality. In L. Pervin \& O. John (Eds.), Handbook of personality (2nd ed., pp. 154-196). New York, NY: Guilford Press.

Bandura, A. (2000). Exercise of human agency through collective efficacy. Current Directions in Psychological Science, 9, 75-78. doi:10.1111/1467-8721.00064

Betz, N. E., \& Voyten, K. K. (1997). Efficacy and outcome expectations influence career exploration and decidedness. Career Development Quarterly, 46, 179-189. doi:10.1002/j.21610045.1997.tb01004.x

Brislin, R. W., (1986). The wording and translation of research instruments. In W. J. Lonner \& J. W. Berry (Eds.), Field methods in cross-cultural research, (pp. 137-164). London, UK: Sage Publications, Inc.

Cheung, R., \& Arnold, J. (2010). Antecedents of career exploration among Hong Kong Chinese university students: Testing contextual and developmental variables. Journal of Vocational Behavior, 76, 25-36. doi:10.1016/j.jvb.2009.05.006

Creed, P. A., Fallon, T., \& Hood, M. (2009). The relationship between career adaptability, person and situation variables, and career concerns in young adults. Journal of Vocational Behavior, 74, 219-229. doi:10.1016/j.jvb.2008.12.004 
Creed, P., Tilbury, C., Buys, N., \& Crawford, M. (2011). The career aspirations and action behaviours of Australian adolescents in out-of-home-care. Children and Youth Services Review, 33, 1720-1729. doi:10.1016/j.childyouth.2011.04.033

Cross, S. E. \& Markus, H. R. (1999). The cultural constitution of personality. In L. Pervin \& O. John (Eds.), Handbook of personality (2 ${ }^{\text {nd }}$ ed., pp. 378-396). New York, NY: Guilford Press.

Flores, L. Y., \& O'Brien, K. M. (2002). The career development of Mexican American adolescent women: A test of social cognitive career theory. Journal of Counseling Psychology, 49, 14-27. doi:10.1037//0022-0167.49.1.14

Fouad, N. A., \& Smith, P. L. (1996). A test of a social cognitive model for middle school students: Math and Science. Journal of Counseling Psychology, 43, 338-346. doi:10.1037/00220167.43.3.338

Fouad, N. A., Kantamneni, N., Smothers, M. K., Chen, Y.-L., Fitzpatrick, M., \& Terry, S. (2008). Asian American career development: A qualitative analysis. Journal of Vocational Behavior, 72, 43-59. doi:10.1016/j.jvb.2007.10.002

Fouad, N. A., Smith, P. L., \& Enochs, L. (1997). Reliability and validity evidence for the Middle School Self-Efficacy Scale. Measurement and Evaluation in Counseling and Development, 30, $17-31$.

Garcia, P. R. J. M., Restubog, S. R. D., Toledano, L. S., Tolentino, L. R., \& Rafferty, A. L. (2012). Differential moderating effects of student- and parent-rated support in the relationship between learning goal orientation and career decision-making self-efficacy. Journal of Career Assessment, 29, 22-33. doi:10.1177/1069072711417162

Greenhaus, J. H. (1971). An investigation of the role of career salience in vocational behavior. Journal of Vocational Behavior, 1, 209-216. doi:10.1016/0001-8791(71)90022-4

Hair, J. F., Black, W. C., Babin, B. J., Anderson, R. E. (2010). Multivariate data analysis. A global perspective. ( $7^{\text {th }}$ ed). New Jersey, NJ: Prentice Hall. 
Hardin, E. E., Leong, F. T. L., \& Osipow, S. H. (2001). Cultural relativity in the conceptualization of career maturity. Journal of Vocational Behavior, 58, 36-52. doi:10.1006/jvbe.2000.1762

Hau, K.-T., \& Marsh, H. W. (2004). The use of item parcels in structural equation modelling: Nonnormal data and small sample sizes. British Journal of Mathematical and Statistical Psychology, 57, 327-351. doi:10.1111/j.2044-8317.2004.tb00142.x

Hirschi, A. (2011). Vocational identity as a mediator of the relationship between core selfevaluations and life and job satisfaction. Applied Psychology: An International Review, 60, 622-644. doi:10.1111/j.1464-0597.2011.00450.x

Hofstede, G., \& Hofstede, G. J. (2005). Cultures and organizations. Software of the mind. (2 ${ }^{\text {nd }}$ ed). New York: McGraw-Hill.

Kelly, K. R., Gunsalus, A-J. C., \& Gunsalus, R. (2009). Social cognitive predictors of the career goals of Korean American students. The Career Development Quarterly, 58, 14-28. doi:10.1002/j.2161-0045.2009.tb00170.x

Klassen, R. M. (2004). Optimism and realism: A review of self-efficacy from a cross-cultural perspective. International Journal of Psychology, 39, 205-230. doi:10.1080/00207590344000330

Kline, R. B. (1998). Principles and practice of structural equation modeling. New York, NY: The Guilford Press.

Lapan, R. T., Shaughnessy, P., \& Boggs, K. (1996). Efficacy expectations and vocational interests as mediators between sex and choice of math/science college majors: A longitudinal study. Journal of Vocational Behavior, 49, 277-291. doi:10.1006/jvbe.1996.04

Lent, R. W. (2005). A social cognitive view of career development and counseling. In S. D. Brown \& R. W. Lent (Eds.), Career development and counseling: Putting theory and research to work (pp. 101-127). New York, NY: Wiley. 
Lent, R. W., Brown, S. D., \& Hackett, G. (1994). Toward a unifying social cognitive theory of career and academic interest, choice, and performance. Journal of Vocational Behavior, 45, 79-122. doi:10.1006/jvbe.1994.1027

Lent, R. W., Brown, S. D., \& Hackett, G. (2000). Contextual supports and barriers to career choice: A social cognitive analysis. Journal of Counseling Psychology, 47, 36-49. doi: 10.1037//00220167.47.1.36

Lent, R. W., Brown, S. D., Brenner, B., Chopra, S. B., Davis, T., Talleyrand, R., \& Suthakaran, V. (2001). The role of contextual supports and barriers in the choice of math/science educational options: A test of social cognitive hypotheses. Journal of Counseling Psychology, 48, 474-483. doi:10.1037/0022-0167.48.4.474

Lent, R. W., Brown, S. D., Schmidt, J., Brenner, B., Lyons, H., \& Treistman, D. (2003). Relation of contextual supports and barriers to choice behavior in engineering majors: Test of alternative social cognitive models. Journal of Vocational Behavior, 50, 458-465. doi:10.1037/00220167.50.4.458

Lent, R. W., Brown, S. D., Sheu, H., Schmidt, J., Brenner, B., Gloster, C. S. . . Treistman, D. (2005). Social cognitive predictors of academic interests and goals in engineering: Utility for women and students at historically Black universities. Journal of Counseling Psychology, 52, 84-92. doi:10.1037/0022-0167.52.1.84

Lent, R. W., Sheu, H., Gloster, C. S., \& Wilkins, G. (2010). Longitudinal test of the social cognitive model of choice in engineering students at historically Black universities. Journal of Vocational Behavior, 76, 387-394. doi:10.1016/j.ivb.2009.09.002.

Lent, R. W., Sheu, H.-B., Singley, D., Schmidt, J. A., Schmidt, L. C., \& Gloster, C. S. (2008). Longitudinal relations of self-efficacy to outcome expectations, interests, and major choice goals in engineering students. Journal of Vocational Behavior, 73, 328-335. doi: 10.1016/j.jvb.2008.07.005 
Leong, F. T. L., \& Serafica, F. C. (1995). Career development of Asian Americans: A research area in need of a good theory. In F. T. L. Leong (Ed.), Career development and vocational behavior of racial and ethnic minorities (pp. 67-102). Hillsdale, NJ: Erlbaum.

Leung, S. A., Hou, Z.-J., Gati, I., \& Li, X. (2011). Effects of parental expectations and culturalvalues orientation on career decision-making difficulties of Chinese university students. Journal of Vocational Behavior, 78, 11-20. doi:10.1016/j.jvb.2010.08.004

Little, T. D., Cunningham, W. A., Shahar, G., \& Widaman, K. F. (2002). To parcel or not to parcel: Exploring the question, weighing the merits. Structural Equation Modeling, 9, 151-173. doi:10.1207/S15328007SEM0902_1

McWhirter, E. H., Rasheed, S., \& Crothers, M. (2000). The effects of high school career education on social cognitive career variables. Journal of Counseling Psychology, 47, 330-341. doi:10.1037/0022-0167.47.3.330

O'Brien, K. M. (1996). The influence of psychological separation and parental attachment on the career development of adolescent women. Journal of Vocational Behavior, 48, 257-274. doi:10.1006/jvbe.1996.0024

Oettingen, G., \& Zosuls, K.M. (2006). Culture and self-efficacy in adolescents. In F. Pajares \& T. Urdan. (Eds.), Self-efficacy beliefs of adolescents. Volume V of adolescence and education (pp. 245-265). Greenwich, CT: Information Age Publishing.

Ojeda, L., \& Flores, L. Y. (2008). The influence of gender, generation level, parents’ education level, and perceived barriers on the educational aspirations of Mexican American high school students. The Career Development Quarterly, 57, 84-95. doi:10.1002/j.21610045.2008.tb00168.x

Restubog, S. L. D., Florentino, A. R., \& Garcia, P. R. J. M. (2010). The mediating roles of career self-efficacy and career decidedness in the relationship between contextual support and persistence. Journal of Vocational Behavior, 77, 186-195. doi:10.1016/j.jvb.2010.06.005 
Reynolds, A. L., \& Constantine, M. G. (2007). Cultural adjustment difficulties and career development of international college students. Journal of Career Assessment, 15, 338-350. doi:10.1177/1069072707301218

Rogers, M. E., \& Creed, P. A. (2011). A longitudinal examination of adolescent career planning and exploration using a social cognitive career theory framework. Journal of Adolescence, 34, 163172. doi:10.1016/j.adolescence.2009.12.010

Rogers, M. E., Creed, P. A., \& Glendon, A. I. (2008). The role of personality in adolescent career planning and exploration: A social cognitive perspective. Journal of Vocational Behavior, 73, 132-142. doi:10.1016/j.jvb.2008.02.002

Schaefers, K. G., Epperson, D. L., \& Nauta, M. M. (1997). Women's career development: Can theoretically derived variables predict persistence in Engineering majors? Journal of Counseling Psychology, 44, 173-183. doi:10.1037/0022-0167.44.2.173

Schumacker, R. E., \& Lomax, R. G. (2010). A beginner's guide to a structural equation modelling. New York, NY: Routledge.

Shea, M., Ma, P. W., \& Yeh, C. J. (2007). Development of a culturally specific career exploration group for urban Chinese immigrant youth. Career Development Quarterly, 56, 62-73. doi:10.1002/j.2161-0045.2007.tb00020.x

Stumpf, S. A., Colarelli, S. M., \& Hartman, K. (1983). Development of the Career Exploration Survey (CES). Journal of Vocational Behavior, 22, 191-226. doi:10.1016/00018791(83)90028-3

Tang, M., Fouad, N. A., \& Smith, P. L. (1999). Asian Americans' career choices: A path model to examine factors influencing their career choices. Journal of Vocational Behavior, 54, 142-157. doi:10.1006/jvbe.1998.1651 
Wang, L.-F., \& Heppner, P. P. (2002). Assessing the impact of parental expectations and psychological distress on Taiwanese college students. The Counseling Psychologist, 30, 582608. doi:10.1177/00100002030004006

Weng, L. J. (2004). Impact of the number of response categories and anchor labels on coefficient alpha and test-retest reliability. Educational and Psychological Measurement, 64, 956-972. doi:10.1177/0013164404268674 
Table 1

Correlations among Variables, Means, Standard Deviations, Scale Range, and Internal Reliability Coefficients (Cronbach’s Alpha); $N=954$

\begin{tabular}{|c|c|c|c|c|c|c|c|c|c|c|c|c|c|c|c|c|c|c|}
\hline Variables & 1 & 2 & 3 & 4 & 5 & 6 & 7 & 8 & 9 & 10 & 11 & 12 & 13 & 14 & $M$ & $S D$ & Range & $\alpha$ \\
\hline T1 Parental expectations & - & .22 & .30 & .30 & .27 & .24 & .21 & .46 & .17 & .19 & .18 & .22 & .20 & .16 & 47.70 & 4.64 & $9-54$ & .80 \\
\hline 2. T1 Congruence & .27 & - & .41 & .42 & .27 & .35 & .37 & .12 & .56 & .29 & .25 & .18 & .26 & .31 & 54.08 & 8.20 & $12-72$ & .87 \\
\hline 3. T1 Self-efficacy & .42 & .52 & - & .59 & .25 & .31 & .36 & .21 & .29 & .48 & .39 & .25 & .31 & .36 & 55.85 & 5.90 & $12-72$ & .75 \\
\hline 4. T1 Outcome expectations & .36 & .48 & .75 & - & .40 & .42 & .37 & .18 & .29 & .40 & .46 & .24 & .29 & .27 & 29.66 & 3.38 & $12-72$ & .79 \\
\hline 5. T1 Career aspirations & .37 & .33 & .60 & .50 & - & .53 & .29 & .24 & .27 & .31 & .35 & .49 & .38 & .30 & 47.68 & 5.38 & $10-60$ & .71 \\
\hline 6. T1 Career planning & .36 & .43 & .69 & .56 & .72 & - & .43 & .19 & .27 & .31 & .30 & .31 & .46 & .34 & 38.65 & 4.33 & $8-48$ & .68 \\
\hline 7. T1 Career exploration & .26 & .42 & .60 & .43 & .36 & .58 & - & .12 & .28 & .36 & .25 & .20 & .30 & .57 & 43.37 & 7.83 & $11-66$ & .83 \\
\hline 8. T2 Parental expectations & .50 & .15 & .29 & .22 & .37 & .25 & .15 & - & .19 & .28 & .26 & .27 & .25 & .19 & 47.52 & 4.87 & $9-54$ & .82 \\
\hline 9. T2 Congruence & .22 & .61 & .37 & .34 & .34 & .36 & .32 & .24 & - & .48 & .43 & .31 & .35 & .39 & 54.45 & 8.22 & $12-72$ & .90 \\
\hline 10. T2 Self-efficacy & .26 & .35 & .63 & .48 & .42 & .44 & .45 & .34 & .58 & - & .64 & .40 & .45 & .50 & 55.07 & 6.41 & $12-72$ & .81 \\
\hline 11. T2 Outcome expectations & .23 & .30 & .50 & .55 & .47 & .41 & .30 & .31 & .51 & .78 & - & .45 & .45 & .34 & 28.92 & 3.49 & $12-72$ & .70 \\
\hline 12. T2 Career aspirations & .30 & .21 & .33 & .30 & .64 & .41 & .25 & .33 & .39 & .50 & .56 & - & .64 & .24 & 46.10 & 6.09 & $10-60$ & .78 \\
\hline 13. T2 Career planning & .28 & .30 & .41 & .39 & .35 & .55 & .36 & .31 & .42 & .55 & .55 & .79 & - & .39 & 37.72 & 4.78 & $8-48$ & .74 \\
\hline 14. T2 Career exploration & .21 & .36 & .47 & .35 & .32 & .45 & .68 & .23 & .45 & .63 & .41 & .32 & .51 & - & 44.41 & 7.75 & $11-66$ & .85 \\
\hline
\end{tabular}

14. T2 Career exploration

Note. Bivariate correlations among diagonal, all significant at $p<.001$. 
Table 2

Path Values in the Standard Causal and the Reciprocal Models

\begin{tabular}{|c|c|c|c|c|}
\hline \multirow[t]{2}{*}{ Time 2 Variables } & \multicolumn{2}{|c|}{ Path Values in the Standard-Causal Model } & \multicolumn{2}{|c|}{ Path Values in the Reciprocal Model } \\
\hline & T1 Variables & Path Coefficient T1 to T2 & T1 Variables & Path Coefficient T1 to T2 \\
\hline \multirow[t]{6}{*}{ Self-efficacy } & Self-efficacy & $.54 * * *$ & Self-efficacy & $.38 * * *$ \\
\hline & Parental expectations & -.01 & Parental expectations & .01 \\
\hline & Congruence & .07 & Congruence & .03 \\
\hline & & & Outcome expectations & $.12 *$ \\
\hline & & & Career planning & .03 \\
\hline & & & Career exploration & $.12 * *$ \\
\hline \multirow[t]{4}{*}{ Outcome expectations } & Outcome expectations & $.31^{* * *}$ & Outcome expectations & $.33 * * *$ \\
\hline & Self-efficacy & $.26^{* * *}$ & Self-efficacy & $.22 *$ \\
\hline & & & Career planning & $.12 *$ \\
\hline & & & Career exploration & -.05 \\
\hline \multirow[t]{5}{*}{ Career aspirations } & Career aspirations & $.59 * * *$ & Career aspirations & $.60 * * *$ \\
\hline & Self-efficacy & -.04 & Self-efficacy & -.03 \\
\hline & Outcome expectations & -.03 & Outcome expectations & -.02 \\
\hline & Parental expectations & $.10^{*}$ & Parental expectations & $.09 *$ \\
\hline & Congruence & .03 & Congruence & .02 \\
\hline \multirow[t]{6}{*}{ Career planning } & Career planning & $.51^{* * *}$ & Career planning & $.53^{* * *}$ \\
\hline & Career aspirations & .05 & Career aspirations & .05 \\
\hline & Self-efficacy & -.06 & Self-efficacy & -.06 \\
\hline & Outcome expectations & .002 & Outcome expectations & .02 \\
\hline & Parental expectations & $.08 *$ & Parental expectations & $.08 *$ \\
\hline & Congruence & .07 & Congruence & .06 \\
\hline \multirow[t]{6}{*}{ Career exploration } & Career exploration & $.52 * * *$ & Career exploration & $.55^{* * *}$ \\
\hline & Career aspirations & $.12^{* *}$ & Career aspirations & $.12 * *$ \\
\hline & Self-efficacy & .14 & Self-efficacy & .11 \\
\hline & Outcome expectations & $-.13^{*}$ & Outcome expectations & -.09 \\
\hline & Parental expectations & -.01 & Parental expectations & -.01 \\
\hline & Congruence & $.10^{*}$ & Congruence & $.08^{*}$ \\
\hline \multirow[t]{2}{*}{ Parental expectations } & - & - & Parental expectations & $.48^{* * *}$ \\
\hline & - & - & Self-efficacy & $.08 *$ \\
\hline \multirow[t]{2}{*}{ Congruence } & - & - & Congruence & $.54 * * *$ \\
\hline & - & - & Self-efficacy & $.12^{* *}$ \\
\hline
\end{tabular}

Note. ${ }^{*} p<.05,{ }^{* *} p<.01,{ }^{* * *} p<.001$. Reverse-direction paths are depicted in boldface. 
Time 1

Time 2

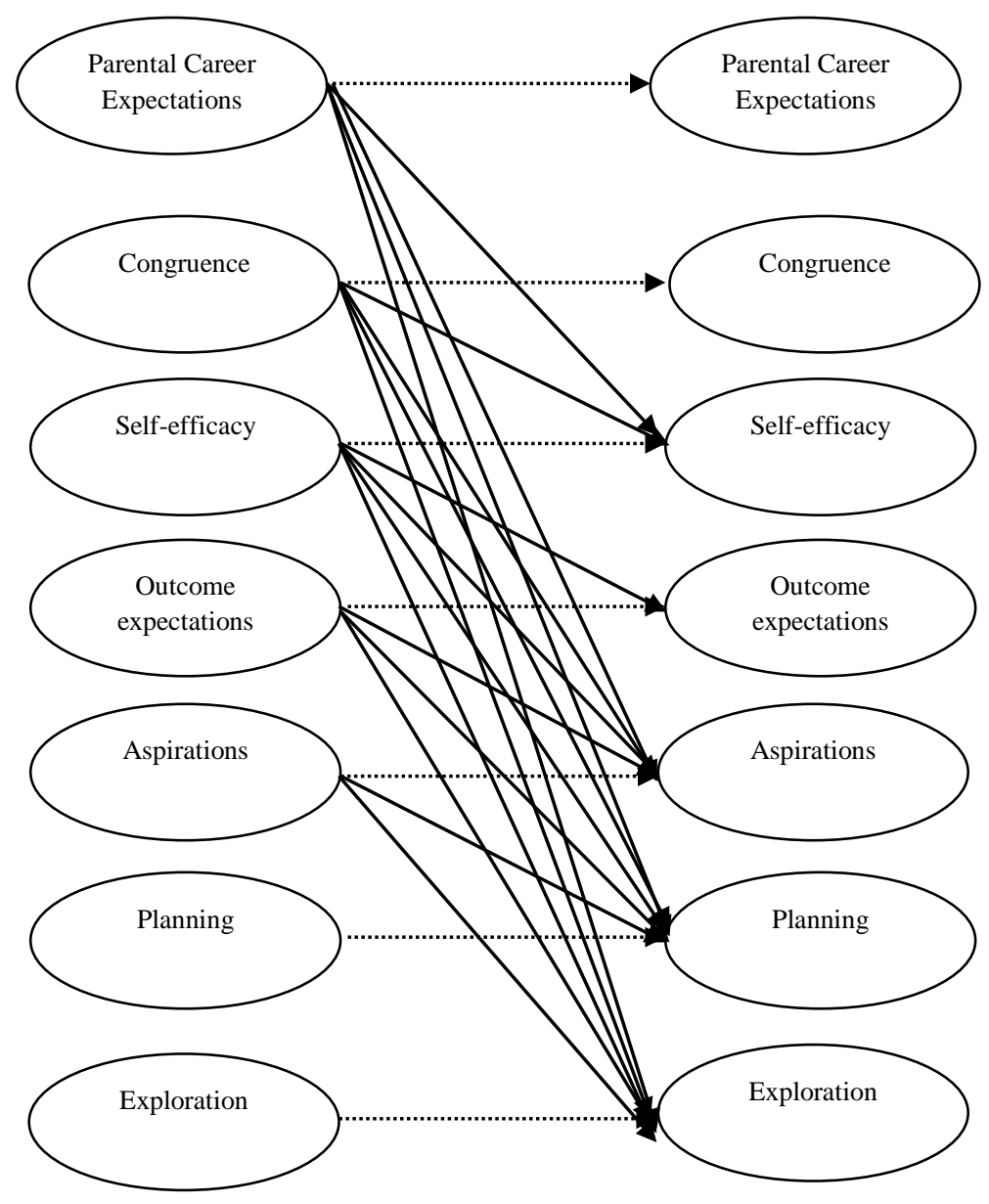

Figure 1. The hypothesised standard-causal model. 
Time 1

Time 2

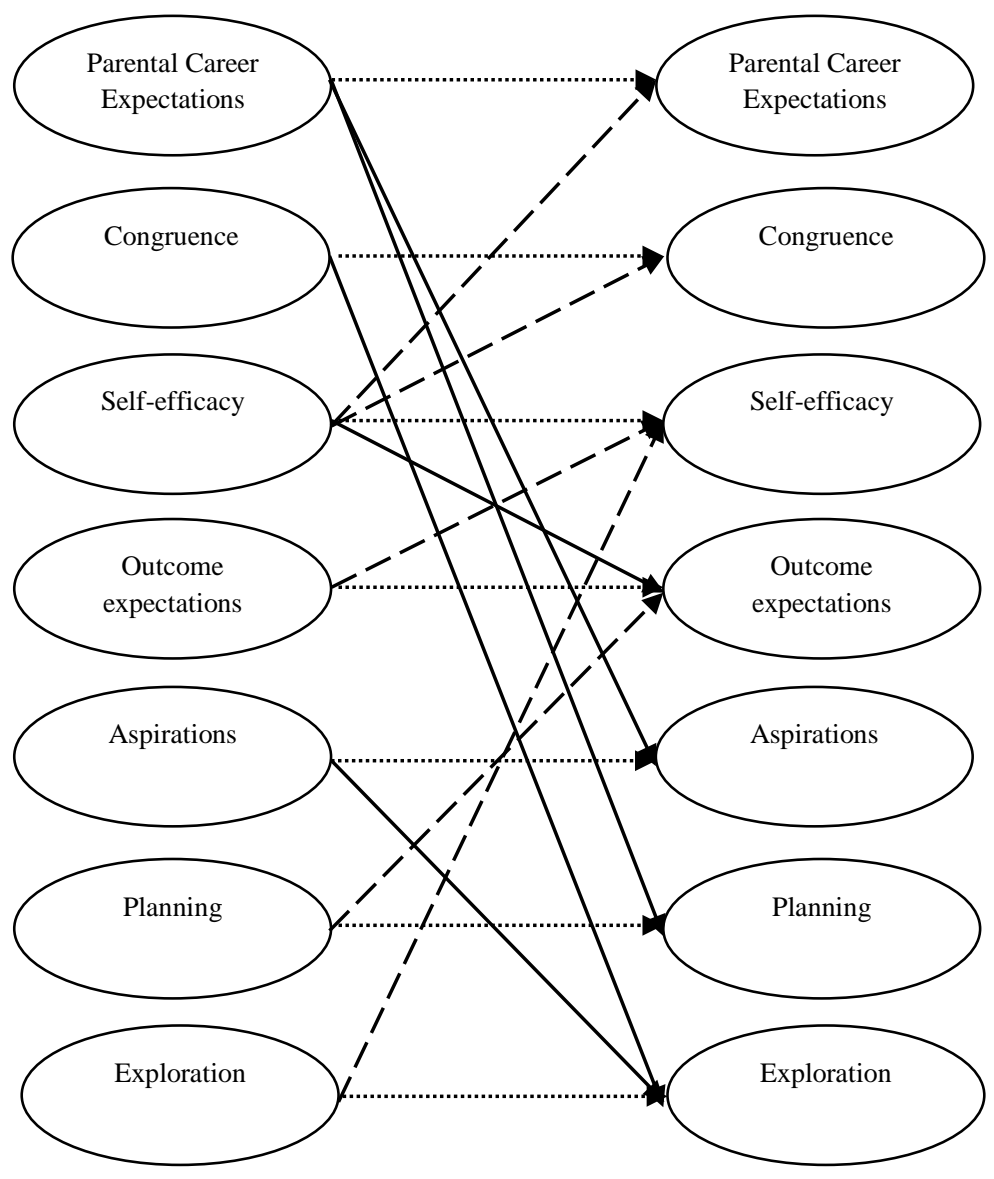

Figure 2. Final reciprocal model. The dotted lines indicate autoregressive paths, the intact lines indicate standard-causal paths, and the dashed lines indicate reverse-direction paths. The non-significant paths have been removed for clarity. 